Training Day in the Autumn attended by two representatives from each of the College Divisions. It is hoped that this experience will enable these members to respond swiftly to their regional media on local problems. This should help the public education activities to develop both centrally and regionally. A report on the activities of the Public Education Department will shortly be published in the Bulletin.
Many of you may know that this is my last year as Registrar of the College. An election is to be held shortly and the results will be announced at the Annual General Meeting of the College in Brighton.

I would like to thank Professor Paykel, his staff and particularly Mary Coburn for their hospitality during our stay in Cambridge.

Prof. R. G. Priest

\title{
Distinction Awards in Psychiatry (England and Wales)
}

A complete list of psychiatrists on Distinction Awards Committees is given below. (see Bulletin, March 1988, 12, 112-113)

\section{Central Committee on Distinction Awards}

College nominated advisers on Awards in the Regions

Northern: Dr K. Schapira; Yorkshire: Dr J. Roberts; Trent: Professor J. E. Cooper; East Anglia: Dr C. P. B. Brook; North West Thames: Dr K. GranvilleGrossman; North East Thames: Dr S. Crown; South West Thames: Professor A. Crisp; South East Thames; Dr T. H. Bewley; Wessex: Professor J. L. Gibbons; Oxford: Dr E. B. O. Smith; South Western: Professor H. G. Morgan; West Midlands: Professor R. Bluglass; Mersey: Professor J. Copeland; North Western: Dr J. Johnson; Wales: Dr D. M. Shaw.

\section{Regional 'Higher' Awards Committee 1988}

\section{Psychiatrist members}

Northern: Dr K. Davison; Yorkshire: Dr J. M. Roberts, Dr I. Berg; Trent: Professor F. A. Jenner, Professor G. E. Roberts; East Anglia: Dr G. E. Berrios; North West Thames: Dr J. Steinert, Professor B. I. Sacks; North East Thames: Professor T. Silverstone; South East Thames: Dr J. Merry, Dr. J. A. Whitehead; South West Thames: Dr A. J. Coppen,
Professor D. Bicknell; Wessex: Professor J. L. Gibbons; Oxford: Professor M. Gelder, Dr D. H. Gath; South Western: Dr C. J. Wardle, Dr J. T. R. Bavin, Dr A. C. Brown; West Midlands: Professor R. S. Bluglass, Dr P. Hall; Mersey: Dr J. S. Madden; North Western: Professor D. C. Taylor, Professor D. P. Goldberg; Wales: Dr M. Tannahill, Dr M. A. Harper.

\section{Regional 'C' Awards Committee 1988}

\section{Psychiatrist members}

Northern: Dr D. A. Stephens, Dr H. Weaving; Yorkshire: Dr S. B. Mahapatra; Dr M. A. Hill; Trent: Dr M. C. Moss; East Anglia: Dr M. O'Brien, Dr J. H. Dowson; North West Thames: Dr M. T. Duff-Miller, Dr G. Kanakaratnam; North East Thames: Dr J. J. Bradley, Dr B. G. Adams; South East Thames: Dr N. Shanmuganathan; South West Thames: Dr S. Craske, Dr A. J. Forrest; Wessex: Dr M. A. RowtonLee, Dr D. H. Dick; Oxford: Professor M. Gelder; South Western: Dr A. C. Fairbairn, Dr Y. Wiley; West Midlands: Dr E. D. Myers, Dr L. I. Liebling, Dr G. Milner; Mersey: Dr J. Warburton, Dr M. W. Forth; North Western: Dr M. D. Cashman, Dr A. Theodossiadis; Wales: Dr D. J. L. Hughes, Dr A. B. Rolfe.

\section{Summer Bank Holiday}

The College will be closed from 7.00 p.m. on Thursday, 25 August until 8.00 a.m. on Tuesday, 30 August 1988. 